\title{
Viviendo con una sola arteria. ¿Es eso posible?
}

\author{
Karla Sue América Hurtado Belizario* \\ Antonio Ángel Skrabonja Crespo** \\ Zoila Rodriguez Urteaga***
}

\author{
Correspondencia \\ Karla Sue América Hurtado Belizario \\ karlahurtadobel @gmail.com
}

* Médico residente de Cardiología. Departamento de Cardiología Clínica. Instituto Nacional Cardiovascular INCOR EsSalud

** Médico asistente de Cardiopediatría. Departamento de Cardiopediatría. Instituto Nacional Cardiovascular INCOR-EsSalud

*** Médico asistente de Cardiología. Servicio de Cardiología no invasiva. Instituto Nacional Cardiovascular INCOR EsSalud

Citar como: Hurtado-Belizario KSA, Skrabonja-Crespo AA, Rodriguez-Urteaga Z. Viviendo con una sola arteria. ¿Es eso posible? Rev Ecocar Pract (RETIC). 2020 (Mar); 3 (1): 62-65. doi: 10.37615/retic.v3n1a17.

Cite this as: Hurtado-Belizario KSA, Skrabonja-Crespo AA, Rodriguez-Urteaga Z. Living with only one artery. Is it possible? Rev Ecocar Pract (RETIC). 2020 (Mar); 3 (1): 62-65. doi: 10.37615/retic.v3n1a17.

\section{Palabras clave \\ $\triangleright$ Tronco arterioso común \\ $\triangleright$ Interrupción de arco aórtico \\ $\triangleright$ Angiotomografía}

\section{RESUMEN}

Se presenta el caso clínico de un paciente de 9 años con tronco arterioso común, de presentación tardía y sin cirugía cardíaca previa, asociado con otras patologías vasculares inusuales y con hipertensión arterial pulmonar severa. Después de 7 años de seguimiento ambulatorio, retorna al hospital por progresión de síntomas. Se realizó estudio con angiotomografía que reveló, adicionalmente, interrupción del arco aórtico (tronco arterioso común tipo A4 por clasificación de Van Praagh), válvula troncal bivalva estenótica y ausencia de la vena cava superior derecha con persistencia de la vena cava superior izquierda drenando al seno coronario. Es el primer caso documentado en Perú.

ABSTRACT
The case of a 9-year-old patient is presented with a common arterial trunk, with late clinical presentation and
without previous cardiac surgery, associated with other unusual vascular pathologies and with severe pulmonary
arterial hypertension. After 7 years of outpatient follow-up, he returns to the hospital due to progression of symp-
toms. A study with angiotomography additionally revealed interruption of the aortic arch (common arterial trunk
type A4 by Van Praagh's classification), stenotic bivalve truncus and absence of the right upper vena cava with
persistence of the left upper vena cava draining to the coronary sinus. This is the first case reported in Peru.

\section{Presentación del caso}

Se presenta el caso de un varón de 9 años, natural de Puno, Perú, con antecedentes de tronco arterioso común (TAC), diagnosticado en la etapa neonatal, que no fue intervenido quirúrgicamente por razones desconocidas.

Fue evaluado por primera vez en la institución de los autores a los 14 meses de edad. En ese momento, estaba taquipnéico, con cianosis leve, peso 7,8 kg, segundo ruido único y soplo sistólico II/NI paraesternal derecho.

En base a la ecocardiografía transtorácica (ETT) bidimensional se diagnosticó TAC tipo I (clasificación de Collet-Edwards), con comunicación interventricular (CIV) subtroncal, estenosis severa de la válvula troncal (VT) y vena cava superior izquierda persistente (VCSIP) al seno coronario.

Se le realizó un cateterismo cardíaco modificándose el diagnóstico a TAC tipo II (clasificación de Collet-Edwards). Además, se documentó hipertensión arterial pulmonar (HTAP) severa con presión arterial pulmonar media (PAPm) de 43 $\mathrm{mmHg}$. Se indicó tratamiento farmacológico a base de furosemida, captopril, espironolactona y sildenafilo. Se valoró en una junta médica que concluyó que existía posibilidad quirúrgica con alto riesgo de mortalidad intraoperatoria. Ante la negativa de los padres y debido a lo complejo del caso, se indicó seguimiento médico.

Retornó al hospital a la edad de 9 años por empeoramiento de su estado clíni$\mathrm{Co}$, en clase funcional NYHA III. El examen físico reveló saturación de oxígeno periférica de 80\%, pobre ganancia ponderal (19 kg), soplo sistólico eyectivo III/ VI en IV espacio intercostal izquierdo y un segundo ruido único aumentado.

Se le realizó una ETT bidimensional con hallazgos similares a la realizada hace 7 años. Un nuevo estudio hemodinámico reveló un aumento en la PAPm de 43 a $52 \mathrm{mmHg}$.

Se decide realizar angiotomografía cardiovascular torácica (angio-TC) que demostró un TAC tipo A4 (clasificación de Van Praagh), con interrupción del arco 
aórtico (IAAo) tipo C, conducto arterioso persistente (CAP) amplio de $17 \mathrm{~mm}$ CIV subtroncal de $23 \mathrm{~mm}$, VT bicúspide con estenosis, dos ostium coronarios yuxtapuestos, dilatación de las cuatro cavidades cardíacas con predominio izquierdo, ausencia de vena cava superior derecha (VCSD) con persistencia de VCSI al seno coronario muy dilatado.

Con los hallazgos de la angio-TC se decidió en junta multidisciplinar realizar el cerclaje de ambas arterias pulmonares con la intención de mejorar su estado clínico y, posteriormente, dependiendo del comportamiento hemodinámico, evaluar la posibilidad de una cirugía reparativa definitiva a mediano plazo.

\section{Estudio por imagen}

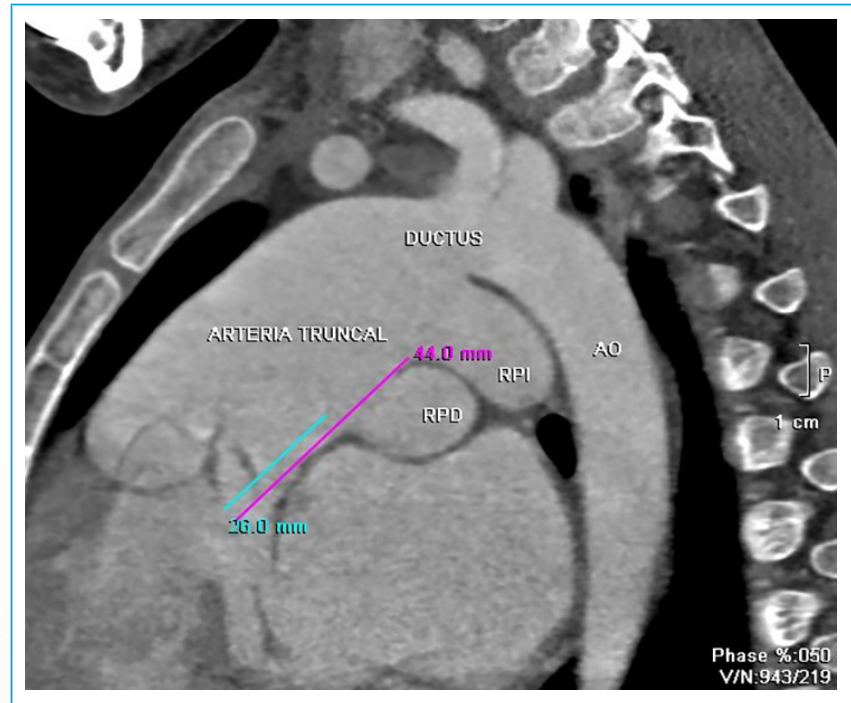

Figura 1. Eje sagital de la angio-TC. Del TAC emergen las ramas pulmonares derecha (RPD) e izquierda (RPI) dilatadas. Éste se continúa con PCA grande y aorta descendente (AO). En posición opuesta al ductus, salen las arterias carótida y subclavia izquierda

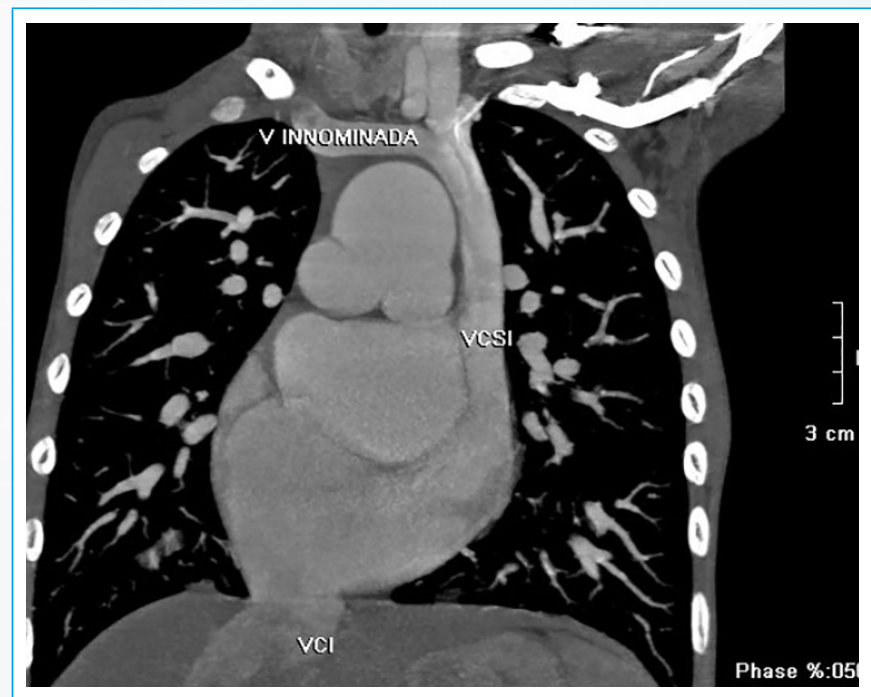

Figura 2. Corte coronal de la angio-TC. La vena (V) innominada derecha drena a una vena cava superior izquierda (VCSI) persistente que desemboca en un seno coronario muy dilatado. Obsérvese la vena cava inferior (VCI) drenando a la aurícula derecha y ausencia de VCS derecha

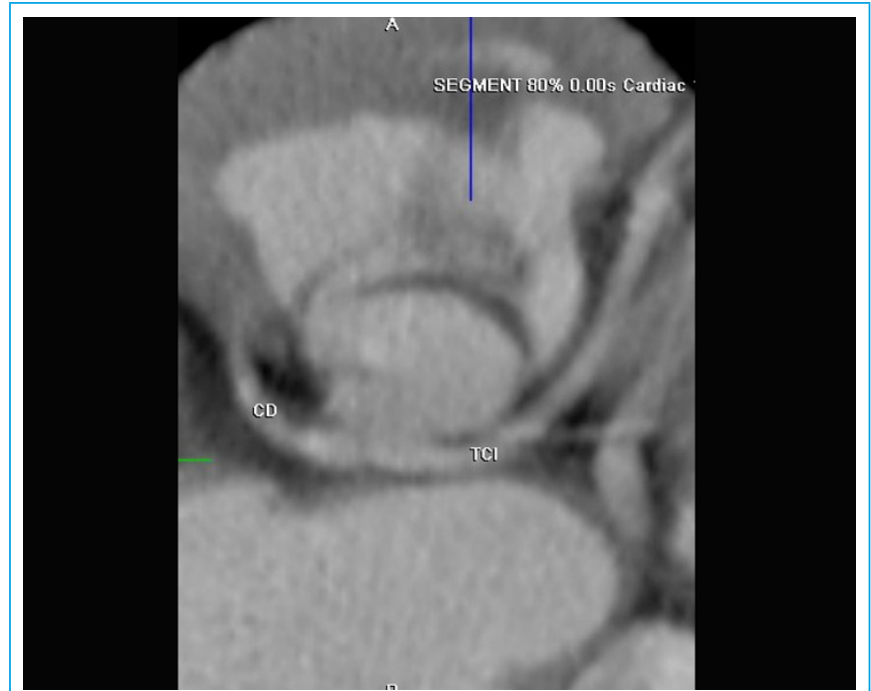

Figura 3. Corte transversal de la angio-TC a nivel de la raíz troncal que muestra una válvula troncal bicúspide

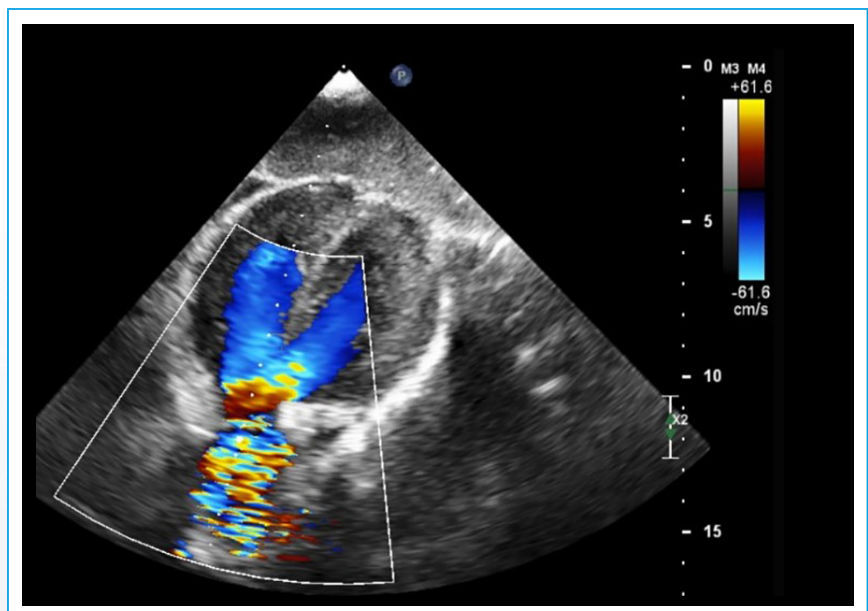

Figura 4. Corte transversal de la angio-TC a nivel del nacimiento de las arterias coronarias, evidenciando 2 ostium coronarios yuxtapuestos de donde emergen la arteria coronaria derecha (CD) y el tronco coronario izquierdo (TCI)

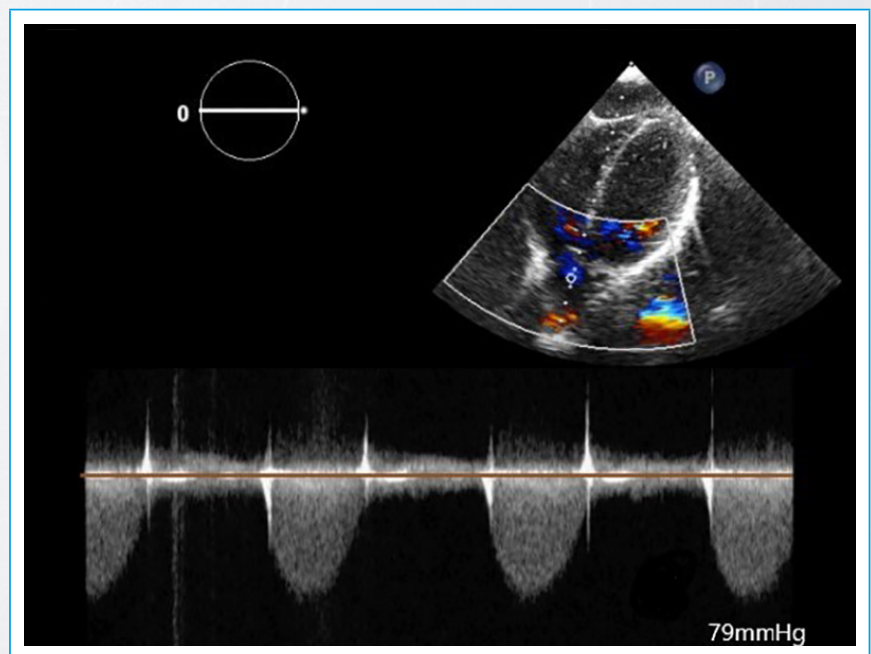

Figura 5. ETT bidimensional con vista de cinco cámaras. Con el Doppler color se observa flujo sanguíneo a nivel de la CIV subtroncal y aceleración sistólica a nivel de la válvula troncal 


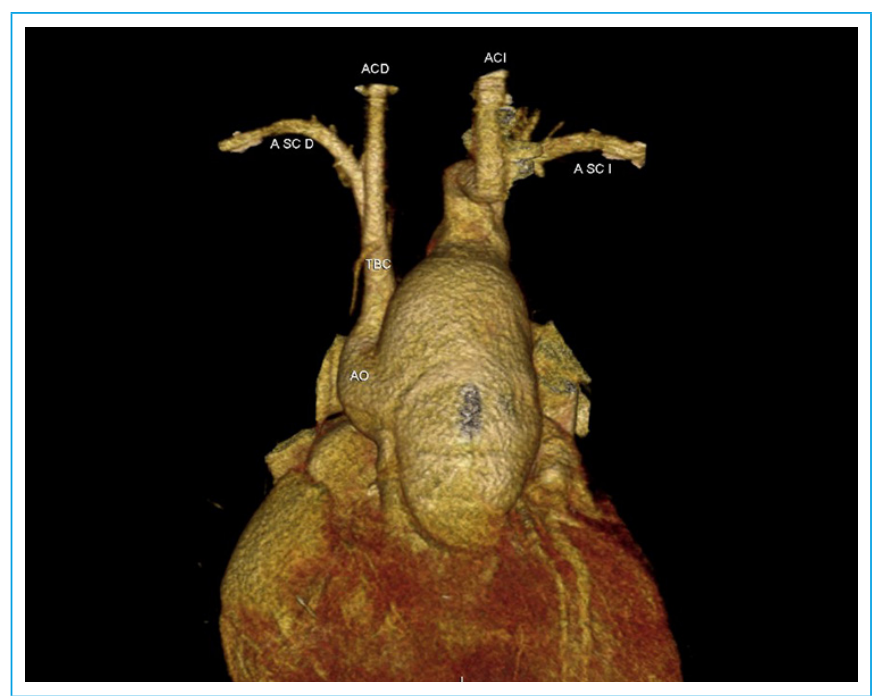

Figura 6. ETT bidimensional con vista de cinco cámaras. El Doppler continuo a nivel de la salida única arterial muestra un gradiente medio de $79 \mathrm{mmHg}$

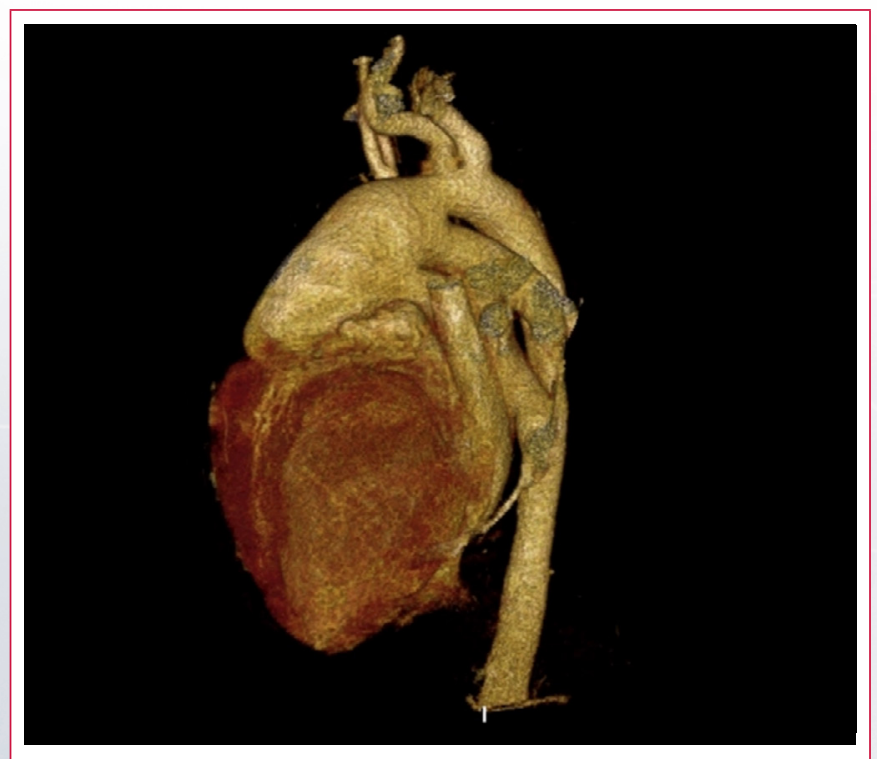

Vídeo 1. Reconstrucción tomográfica completa en tres dimensiones del tronco arterioso común con interrupción del arco aórtico tipo C
La PVCSI con ausencia de la VCSD ocurre en 0,09 a 0,13\% de pacientes con

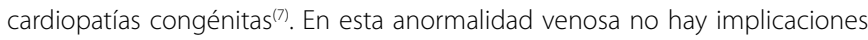
hemodinámicas, pero sí toma importancia cuando se vaya a obtener una vía venosa central utilizando la vena yugular interna.

La presencia de HTAP severa y la relativa edad avanzada del paciente constituyen factores de mal pronóstico. Desde un inicio se planteó la posibilidad quirúrgica de cerclaje pulmonar bilateral con el fin de disminuir las presiones pulmonares y, luego, planificar la cirugía reparativa definitiva. Por negativa de los padres y lo complejo de su cardiopatía no se realizó.

Es muy inusual que un paciente con TAC sobreviva sin cirugía hasta los 9 años. Los factores que probablemente han favorecido la supervivencia del paciente son un PCA no restrictivo, resistencias vasculares pulmonares elevadas y ausencia de insuficiencia de VT. Otro dato interesante es que, durante este tiempo, el paciente ha vivido en Puno, ciudad de los Andes peruanos, ubicada a 3.827 metros sobre el nivel del mar. Es posible que exista una relación entre la altura y el comportamiento no habitual de esta compleja cardiopatía en el paciente. Sin embargo, no se tiene conocimiento que se haya descrito en la literatura un TAC con este tipo de asociación. Sin embargo, existe una relación de tipo parabólica entre la presión pulmonar y la altura del lugar de nacimiento, según el estudio realizado por Cruz-Jibaja et al.(5). De lo expuesto se puede desglosar una interrogante: iserá que en este paciente la altura extrema ha permitido su supervivencia más allá de lo habitual para una patología tan severa?

\section{Conclusión}

Este caso resalta la importancia del uso de imágenes cardíacas de forma multimodal para lograr un diagnóstico preciso de una cardiopatía compleja como el TAC. Esta forma de estrategia ayuda a identificar la anatomía y la fisiopatología de las cardiopatías congénitas de una manera objetiva y certera. Esta información es crucial para decidir cuál es la mejor estrategia terapéutica para seguir.

\section{Ideas para recordar}

- EI TAC es una cardiopatía congénita severa poco frecuente con una pobre supervivencia si no se diagnostica y opera óptimamente antes de los 6 meses de edad.

- EITAC, como muchas otras cardiopatías congénitas, puede tener otras anormalidades cardiovasculares asociadas de importancia clínica.

- El abordaje multimodal de imágenes es indispensable en la evaluación de las cardiopatías congénitas.

\section{Bibliografía}

EI TAC pertenece a la familia de las anomalías conotroncales y se caracteriza por un tronco arterial solitario que emerge de la base del corazón y da origen a las arterias coronarias, pulmonares y al menos a una arteria braquiocefálica ${ }^{(1)}$. Esta entidad representa cerca del $1 \%$ de las cardiopatías congénitas ${ }^{(2)}$. La mayor variabilidad anatómica se encuentra en el arco aórtico. Una patología asociada importante es la IAAo $(11 \text { a 19\% })^{(2)}$ y tiene que haber un PCA que permita que haya flujo sanguíneo en la aorta descendente. La VT tiene 3 valvas en dos terceras partes de los pacientes; en el resto, puede haber de 2 a 6 valvas ${ }^{(2)}$. No es infrecuente que haya insuficiencia de la VT, causada por engrosamiento y displasia o por prolapso debido a la dilatación de la raíz troncal. Es inusual la presencia de estenosis de la $\mathrm{V}^{(1,2)}$. Springer International Publihing. Switzerland. 2016.

2. Zampi JD. Moss and Adams' Heart Disease in Infants, Children, and Adolescents: Including the Fetus and Young Adult [Internet]. JAMA 2008. 300: 2676.

3. Pierpont MEM, Gobel JW, Moller JH, Edwards JE. Cardiac malformations in relatives of children with truncus arteriosus or interruption of the aortic arch. Am J Cardiol 1988; 61 (6): 423-427.

4. Bohuta L, Hussein A, Fricke TA, et al. Surgical repair of truncus arteriosus associated with interrupted aortic arch: Long-term outcomes. Ann Thorac Surg 2011; 91 (5): 1473-1477.

5. Cruz-Jibaja J. Correlation Between Pulmonary Artery Pressure and Level of Altitude. CHEST J 1964; 46 (4): 446. 
6. Toma D, Șuteu CC, Togănel R. Favorable Postoperative Evolution after Late Surgical Repair of Truncus Arteriosus Type I: A Case Report. JIM 2018; 3 (50): $110-113$
7. Azeem S, et al. Persistent left superior vena cava with absent right superior vena cava: Review of the literature and clinical implications. Echocardiography 2014; 31: 674-679. 